\title{
Optimization of Thermal and Mechanical Properties in Nanoporous Materials
}

\author{
Iwan Sumirat, Noriyuki Yamamoto* and S. Shimamura* \\ Neutron Scattering Laboratory, PTBIN - BATAN, Puspiptek Serpong, Tangerang, Banten, Indonesia \\ *Faculty of Engineering, Yamaguchi University, Ube 755-8611, Japan \\ E-mail: simamura@yamaguchi-u.ac.jp
}

\begin{abstract}
We have studied theoretically thermal and mechanical properties of nanoporous materials. The thermal conductivity has been estimated with special attention to phonon scattering by nanometer-sized pores. It is shown that the thermal conductivity of a material having several nanometer-sized pores decreases steeply with increasing porosity. The bulk modulus of model nanoporous materials has been calculated using an interatomic potential. It is shown that the bulk modulus of a material having several nanometer-sized pores decreases moderately with increasing porosity. From our theoretical consideration we discuss how we optimize thermal and mechanical properties of nanoporous materials.

Key words: nanoporous materials, thermal conductivity, bulk modulus, porosity, pore-size
\end{abstract}

\section{INTRODUCTION}

The existence of pores in materials causes decreases in the thermal conductivity and the modulus of elasticity. Both the quantities decrease with increasing porosity. The porosity dependences of these quantities have been investigated for many porous materials having micrometer-sized pores [1]. The porosity is the most important factor affecting properties of microporous materials. The pore size does not seem to appreciably influence properties of microporous materials.

The latest developments of nanotechnology have opened up the possibilities of tailor-made nanoporous materials. At present, there is little quantitative data on the porosity dependences of thermal and mechanical properties of nanoporous materials. Also there are almost no theoretical studies on the porosity dependence of those properties. Moreover very little is known about the influence of pore-size on properties of nanoporous materials.

The development of new porous materials having lower thermal conductivity and higher mechanical strength is required for practical purposes such as thermal insulation materials. In this study we study theoretically the porosity and pore-size dependences of thermal and mechanical properties of nanoporous materials. The thermal conductivity and the bulk modulus are investigated for materials having isolated nanometer-sized pores. On the basis of our investigations, we will discuss how we can optimize thermal and mechanical properties of nanoporous materials.

\section{THERMAL CONDUCTIVITY}

There are many experimental data on the thermal conductivity of various porous materials [1-4], but data on the porosity dependence over the wide range of porosity are very scarce. Furthermore very little is known about the pore-size dependence of the thermal conductivity. Many models for the porosity dependence of the thermal conductivity based on theories of twocomponent materials have been proposed [1, 4-7]. Nevertheless, those models have not taken account of the effect of pore size on the thermal conductivity. Therefore we here present an expression for the thermal conductivity which depends on pore size of nanoporous materials.

We consider a material having isolated nanometersized pores. The thermal conductivity $\kappa$ of insulating solids is expressed as

$$
\kappa=\frac{1}{3} C v l
$$

where $C$ is the heat capacity per unit volume, $v$ is the phonon velocity, and $l$ is the phonon mean free path. We inquire about the dependences of $C, v$ and $l$ on porosity at room temperature.

The heat capacity per unit volume $C$ is reduced by the existence of pores because the heat capacity of pores is negligibly small compared with that of the solid phase. Thus we can assume that $C$ in porous materials depends on porosity $\phi$ as

$$
C=C_{0}(1-\phi),
$$

where $C_{0}$ is the heat capacity at $\phi=0$.

The phonon velocity $v$ is determined by the interatomic interaction. We here assume roughly that $v$ is not remarkably affected by the presence of pores as

$$
v=v_{0},
$$

where $v_{0}$ is the phonon velocity at $\phi=0$. This assumption is reasonable for small porosity. 
The phonon mean free path $l$ in porous materials must be estimated by considering different processes of phonon scattering. We notice that a nanometer-sized pore contributes to phonon scattering as a scattering center, while a micrometer-sized pore contributes to phonon scattering as the boundary of solid phase. Thus we divide the processes of phonon scattering in nanoporous materials into scattering by nanometer-sized pores and scattering by the others. The latter scattering corresponds to scattering at $\phi=0$, that is, scattering by other phonons and lattice imperfections such as defects and impurities.

When $\tau_{0}$ and $\tau_{\mathrm{p}}$ are the average times between scatterings at $\phi=0$ and by nanometer-sized pores, respectively, the average time between phonon scatterings, $\tau$ is given by $\tau^{-1}=\tau_{0}^{-1}+\tau_{\mathrm{p}}{ }^{-1}$. Since $l=v \tau=$ $v_{0} \tau$, it holds that $l^{-1}=l_{0}^{-1}+l_{\mathrm{p}}^{-1}$, where $l_{0}$ is the phonon mean free path at $\phi=0$ and $l_{\mathrm{p}}$ is the phonon mean free path of phonon-pore scattering. Thus we have

$$
l=l_{0}\left(1+\frac{l_{0}}{l_{\mathrm{p}}}\right)^{-1} .
$$

We consider a nanoporous material in which isolated spherical or cubic pores are distributed at random, as shown in Fig. 1. When there are $N$ pores of pore-size $d$ in a material of volume $V$, the total volume of all pores $V_{\mathrm{p}}$ is given approximately by $V_{\mathrm{p}}=N d^{3}$. Thus the porosity is $\phi=V_{\mathrm{p}} / V=N d^{3} / V$. The material volume per pore $V / N$ is then written in terms of pore-size $d$ and porosity $\phi$ by

$$
\frac{V}{N}=\frac{d^{3}}{\phi} .
$$

On the other hand, the material volume per pore can also be written approximately as

$$
\frac{V}{N}=D^{3}
$$

using the mean distance $D$ between adjacent pores as shown in Fig. 1. From Eqs. (5) and (6), we have

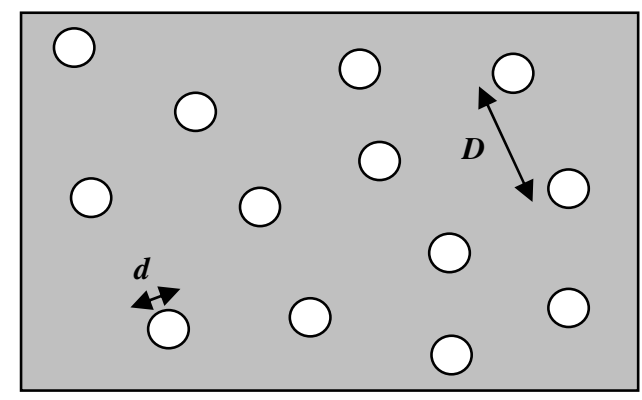

Fig. 1 Schematic view of a model nanoporous material with pore size $d$ and mean distance $D$.

$$
D=\frac{d}{\phi^{1 / 3}} .
$$

The phonon mean free path $l_{\mathrm{p}}$ of phonon-pore scattering is almost equal to the mean distance between adjacent pores $D$. By substituting $l_{\mathrm{p}}$ given by the right side of Eq. (7) into Eq. (4), we have

$$
l=\frac{l_{0}}{1+\frac{l_{0}}{d} \phi^{1 / 3}}
$$

From the expressions for $C, v$ and $l$ of Eqs. (2), (3) and (8), the thermal conductivity $\kappa$ of Eq. (1) is expressed as

$$
\frac{\kappa}{\kappa_{0}}=\frac{1-\phi}{1+\frac{l_{0}}{d} \phi^{1 / 3}},
$$

where $\kappa_{0}$ is the thermal conductivity at $\phi=0$, that is, $\kappa_{0}=(1 / 3) C_{0} v_{0} l_{0}$. The thermal conductivity of nanoporous materials not only depends on porosity $\phi$, but also depends on the ratio of the pore size $d$ to the phonon mean free path $l_{0}$ at $\phi=0$.

The porosity dependences of the thermal conductivity ratio $\kappa / \kappa_{0}$ for $d / l_{0}=10,2$ and 1 are shown in Fig. 2. At the same porosity, the mean distance between adjacent pores becomes shorter when the pore size becomes smaller, as seen in Eq. (7). When $d / l_{0}=10$, the mean distance between adjacent pores is long compared with the phonon mean free path at zero-porosity. Therefore the effect of phonon scattering by pores is not appreciable. When $d / l_{0}=2$, the phonon-pore scattering seems to have a significant effect on the decrease in the thermal conductivity. When $d / l_{0}=1$, the phonon scattering by pores dominates the phonon mean free path

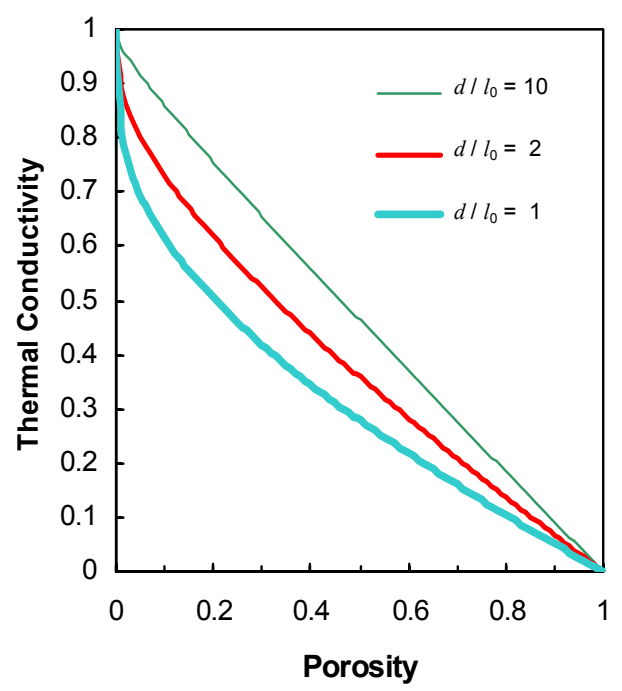

Fig. 2 Porosity dependences of the thermal conductivity for different pore sizes $\left(d\right.$ : pore size, $l_{0}$ : phonon mean free path at zero porosity). 
as well as the scattering by other phonons and lattice imperfections. In this case, the thermal conductivity of nanoporous materials decreases steeply with increasing porosity, as shown in Fig. 2.

\section{BULK MODULUS}

There are many experimental data on mechanical properties of various porous materials [1, 8-13], but data on the porosity dependence over the wide range of porosity are also scarce. Furthermore very little is known about the pore-size dependence of mechanical properties. Munro [14] has proposed an expression for the porosity dependence of the bulk modulus of porous ceramics using the effective medium theory. In the effective medium theory, a porous material is replaced by a uniform medium of zero porosity and low density. The pore-size dependence of the bulk modulus has not been incorporated in the theory. Therefore we here present an expression for the bulk modulus which depends on pore size of nanoporous materials.

We consider a material having isolated nanometersized pores. Pores are assumed to be distributed periodically, as shown in Fig. 3. The bulk modulus can be calculated by using an interatomic potential. We use a simple form for the potential energy of two atoms at separation $r$ as

$$
U=-\frac{\alpha}{r^{\mathrm{n}}}+\frac{\beta}{r^{\mathrm{m}}}
$$

Then the bulk modulus is represented by

$$
B=V_{\mathrm{eq}}\left(\frac{d^{2} E}{d V^{2}}\right)_{V=V_{\mathrm{eq}}}=\frac{\mathrm{nm} E_{\mathrm{eq}}}{9 V_{\mathrm{eq}}},
$$

where

$$
\left(\frac{d E}{d V}\right)_{V=V_{\mathrm{eq}}}=0 .
$$

Here $V_{\text {eq }}$ is the equilibrium volume of a material, $E$ is the total potential energy between atoms, and $E_{\mathrm{eq}}$ is the cohesive energy, that is, the magnitude of $E$ at the equilibrium volume.

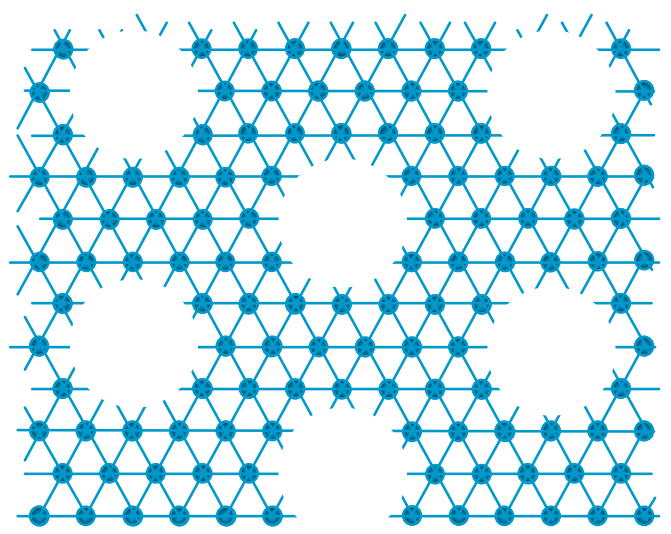

Fig. 3 A model nanoporous material for calculating the bulk modulus.
Obtaining the expressions for $E_{\text {eq }}$ and $V_{\text {eq }}$ from the interatomic potential of Eq. (10), we can have an expression for the ratio $B / B_{0}$, where $B$ is the bulk modulus of a porous material and $B_{0}$ is that of the zeroporosity material. The expression is

$$
\frac{B}{B_{0}}=\left(\frac{A_{\mathrm{n}} A_{\mathrm{m}}^{(0)}}{A_{\mathrm{n}}^{(0)} A_{\mathrm{m}}}\right)^{\left(\frac{\mathrm{n}+3}{\mathrm{~m}-\mathrm{n}}\right)} \frac{A_{\mathrm{n}}}{A_{\mathrm{n}}^{(0)}} .
$$

Here $A_{\mathrm{n}}{ }^{(0)}\left(A_{\mathrm{m}}{ }^{(0)}\right)$ for the zero-porosity material is given by

$$
A_{\mathrm{n}}^{(0)}=\sum_{j}^{j \neq i} \frac{1}{c_{i j}{ }^{\mathrm{n}}},
$$

where $c_{i j}$ is the distance between atoms $i$ and $j$ in the unit of the nearest-neighbor distance for the zero-porosity material; $A_{\mathrm{n}}{ }^{(0)}\left(A_{\mathrm{m}}{ }^{(0)}\right)$ is independent of $i$. Also $A_{\mathrm{n}}\left(A_{\mathrm{m}}\right)$ in Eq. (13) is for a porous material given by

$$
A_{\mathrm{n}}=\frac{1}{N} \sum_{i} \sum_{j}^{j \neq i} \frac{1}{c_{i j}{ }^{\mathrm{n}}},
$$

where $N$ is the number of atoms and the summation includes all atoms except $j=i$.

The calculation of $A_{\mathrm{n}}{ }^{(0)}\left(A_{\mathrm{m}}{ }^{(0)}\right)$ for zero-porosity is straightforward for different crystal lattices. The calculation of $A_{\mathrm{n}}\left(A_{\mathrm{m}}\right)$ for a porous material can be performed if pores are distributed periodically as shown in Fig. 3. Because a porous material loses atoms inside pores, $A_{\mathrm{n}}<A_{\mathrm{n}}{ }^{(0)}$ and $A_{\mathrm{m}}<A_{\mathrm{m}}{ }^{(0)}$. The calculations have been performed for $\mathrm{n}=6$ and $\mathrm{m}=12$ as the LennardJones potential.

Figure 4 shows the porosity dependences of the bulk modulus ratio $B / B_{0}$ for different pore-sizes in the triangular lattice. The number following $\mathrm{P}$ in Fig. 4 is the number of lost atoms per pore. The bulk modulus of

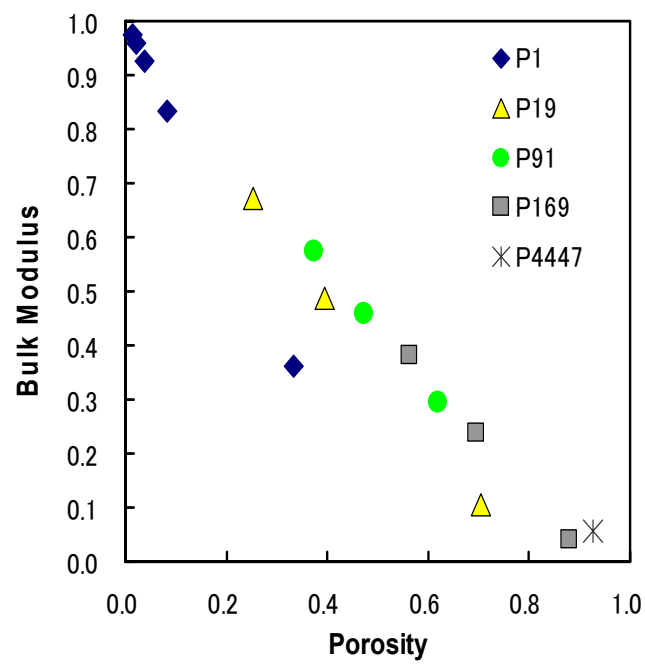

Fig. 4 Porosity dependences of the bulk modulus for different pore sizes. The number following $\mathrm{P}$ is the number of lost atoms per pore. 
a material having smaller-sized pores drops more at the same porosity because more interatomic bonds are lost for a material having smaller-sized pores at the same porosity.

\section{OPTIMIZATION OF PROPERTIES}

From our studies, we will discuss the possibilities of developing nanoporous materials which have lower thermal conductivity and higher modulus of elasticity.

Figure 2 shows that a decrease in pore-size results in a decrease in the thermal conductivity. This is attributed to a decrease in the phonon mean free path through phonon scattering by many small pores. The thermal conductivity decreases steeply with increasing porosity when pore-size is near to the phonon mean free path for zero-porosity $l_{0}$. The value of $l_{0}$ is several nanometers at room temperature for typical insulating crystals. Thus nanoporous materials with the pore-size of several nanometers are favorable for thermal insulation materials.

Figure 4 shows that a decrease in pore-size results also in a decrease in the bulk modulus. This is ascribed to a decrease in interatomic bonds which dominate the bulk modulus. The decrease in the bulk modulus becomes remarkable when pore-size becomes small. However it should be noted that there is only a slight difference among the pore-size dependences for different pore-sizes except an atomic-sized pore such as a vacancy, as shown in P1 of Fig. 4. Therefore we expect that the modulus of elasticity as the bulk modulus in nanoporous materials does not depend appreciably on the pore-size more than one nanometer.

From the above-mentioned pore-size dependences of the thermal conductivity and the bulk modulus, we suggest that a porous material having pores of several nanometers in size is a promising material having considerable thermal insulation and moderate stiffness.

The distribution of pores possibly influences thermal and mechanical properties of nanoporous materials. If pores are distributed nonuniformly, the modulus of elasticity and also the material strength will decrease because the local regions of high pore-density causes low resistance to deformation and fracture. The nonuniform distribution of pores also will cause low thermal conductivity for the same reason as low thermal conductivity in disordered solids. Therefore the pore distribution may be an important factor for optimizing thermal and mechanical properties of nanoporous materials.

In the estimation of the thermal conductivity ratio of Eq. (9), we have assumed that the phonon velocity in a porous material is the same as that in the zero-porosity material. In the calculation of the bulk modulus ratio of Eq. (13), we have used a short-range interatomic potential with $n=6$ and $m=12$ in Eq. (10). The effect of the phonon velocity and the interatomic potential will be left for future study.

In summary we have studied theoretically the porosity and pore-size dependences of the thermal conductivity and the bulk modulus in model nanoporous materials having isolated pores. From our studies we expect that nanoporous materials having pores of several nanometers in size are favorable for thermal insulation materials with moderate stiffness.

\section{REFERENCES}

[1] R. W. Rice, Porosity of Ceramics (Marcel Dekker, New York, 1998).

[2] A. Jain, S. Rogojevic, S. Ponoth, W. N. Gill, J. L. Plawsky, E. Simonyi, S-T. Chen and P. S. Ho, J. Appl. Phys., 91 [5], 3275-3281 (2002).

[3] C. Hu, M. Morgen, P. S. Ho, A. Jain, W. N. Gill, J. L. Plawsky and P. C. Wayner, Jr., Appl. Phys. Lett., 77 [1], 145-147 (2000).

[4] J. Liu, D. Gan, C. Hu, M. Kiene, P. S. Ho, W. Volksen and R. D. Miller, Appl. Phys. Lett., 81 [22], 4180-4182 (2002).

[5] G. Gesele, J. Linsmeier, V. Drach, J. Fricke, and R. Arens-Fischer, J. Physics D, 30, 2911 (1997).

[6] D. W. Song, W. N. Shen, B. Dunn, C. D. Moore, M. S. Goorsky, T. Radetic, R. Gronsky and G. Chen, Appl. Phys. Lett., 84 [11], 1883 (2004).

[7] J. C. Maxwell-Garnet, Philos. Trans. R. Soc. London, 203, 385 (1904).

[8] I. C. van den Born, A. Santen, H. D. Hoekstra and J. Th. M. De Hosson, Phys. Rev. B, 43 [4], 3794-3796 (1991).

[9] N. Ramakrishnan and V. S. Arunachalam, J. Am. Ceram. Soc., 76 [11], 2745-2752 (1993).

[10] K. Tanaka, Y. Akiniwa and T. Ito, Mater. Sci. Res. Int., 6 [1], 49-54 (2000).

[11] R. N. Das, A. Bandyopadhyay and S. Bose, J. Am. Ceram. Soc., 84 [10], 2421-2423 (2001).

[12] Zhen-Yan Deng, T. Fukasawa, M. Ando, Guo-Jun Zhang and T. Ohji, J. Am. Ceram. Soc., 84 [11], 2638-2644 (2001).

[13] Jian-Feng Yang, T. Ohji, S. Kanzaki, A. Diaz and S. Hampshire, J. Am. Ceram. Soc., 85 [6], 1512-1516 (2002).

[14] R. G. Munro, J. Am. Ceram. Soc., 84 [5], 1190-1192 (2001).

(Received December 10, 2008; Accepted May 17, 2009) 